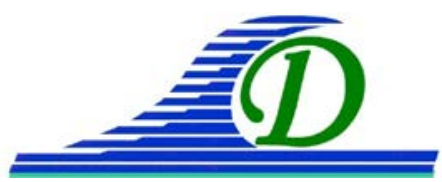

XIII ${ }^{\text {èmes }}$ Journées Nationales Génie Côtier - Génie Civil Dunkerque, 2-4 juillet 2014

DOI:10.5150/jngcgc.2014.099 @ Editions Paralia CFL

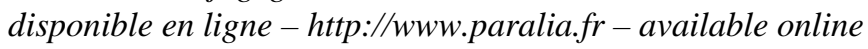

\title{
Modélisation de la submersion marine lors de la tempête Johanna (2008) à Gâvres (Morbihan) : phénomène de franchissement en zone urbaine
}

\section{Sylvestre LE ROY ${ }^{1}$, Rodrigo PEDREROS ${ }^{1}$, Camille ANDRE ${ }^{1,2}$, François PARIS ${ }^{1}$, Sophie LECACHEUX ${ }^{1}$, Fabien MARCHE ${ }^{3}$, Charlotte VINCHON $^{1}$}

1. BRGM, Direction Risques et Prévention, 3 avenue Claude Guillemin, BP 36009, 45060 Orléans Cedex 2, France. s.leroy@brgm.fr ; r.pedreros@brgm.fr ; f.paris@brgm.fr; s.lecacheux@brgm.fr ; c.vinchon@brgm.fr

2. LETG-Brest Géomer, UMR 6554 CNRS, Université de Bretagne Occidentale, Institut Européen de la Mer, Place Nicolas Copernic, 29280 Plouzané, France. camilleg.andre@gmail.com

3. I3M (Univ. de Montpellier 2) - INRIA LEMON, CC051, 34000 Montpellier, France. Fabien.Marche@math.univ-montp2.fr

\section{Résumé :}

La méthodologie présentée dans le présent article vise à simuler de manière la plus réaliste possible les franchissements par paquets de mer et la submersion qui en résulte en milieu urbain. Elle s'appuie sur des simulations régionales à locales de niveaux d'eau et de vagues pour établir des conditions de forçage pour un modèle à résolution de phase ("vague-à-vague") utilisant des schémas numériques adaptés. Ce modèle est alors utilisé sur un Modèle Numérique d’Elévation (MNE), intégrant les bâtiments, de manière à simuler simultanément le franchissement et la propagation de l'inondation.

Cette méthodologie a été appliquée avec le modèle SURF-WB à la submersion survenue à Gâvres (Morbihan) lors de la tempête Johanna (10 mars 2008). Les résultats obtenus s'avèrent tout à fait cohérents avec les observations disponibles (zones de franchissement, étendue de l'inondation, hauteurs d'eau et chronologie). Cette méthode permet d'améliorer la qualité des résultats en milieu urbain par rapport aux approches plus classiques, notamment vis-à-vis de la vitesse des courants à terre. Le développement rapide et récent de ce type de modèle devrait permettre la généralisation de ce type d'approche dans les années à venir. Ce travail a été réalisé dans le cadre du projet JOHANNA (partenariat BRGM-UBO, cofinancé par la Fondation MAIF).

Mots-clés : Submersion marine, Tempête, Vague, Surcote, Franchissement par paquets de mer, Modélisation 2D, Zone urbaine.

\section{Introduction}

La submersion marine par franchissement reste aujourd'hui une problématique mal maitrisée dans le domaine des risques côtiers. Les méthodes actuellement mises en œuvre s’appuient généralement sur des formulations empiriques du débit franchissant, 


\section{Thème 7 - Risques côtiers}

qui permettent le forçage de modèles hydrodynamiques pour simuler l'inondation ; ces approches présentent toutefois un certain nombre de limites, notamment vis-à-vis de leur domaine d'application (elles supposent généralement une configuration idéalisée de la côte et de la bathymétrie qui peut n'être pas compatible avec une situation réelle) et par le fait qu'elles lissent l'effet de chaque vague en un débit moyen continu. Les récents développements apportés aux modèles à résolution de phase permettent désormais d'envisager des simulations réalistes du franchissement, tout en simulant la submersion dans un milieu urbain à la topographie complexe (bâtiments).

Le présent article propose une méthodologie de simulation, de l'échelle régionale à l'échelle très locale, qui permet de simuler de manière réaliste à la fois le franchissement par paquets de mer et la submersion qui en résulte en milieu urbanisé. Cette méthodologie est mise en œuvre et validée sur la submersion survenue à Gâvres (Morbihan) lors de la tempête Johanna en 2008.

\section{Stratégie de modélisation}

La méthodologie proposée (figure 1) s’appuie sur des simulations des niveaux d'eau à l'échelle régionale et côtière, puis des états de mer à l'échelle côtière et locale, afin d'élaborer un forçage réaliste pour un modèle "vague-à-vague" en 2 dimensions capable de simuler à la fois le franchissement des vagues et l'inondation en milieu urbain. Cette méthodologie a été mise en œuvre pour reconstituer les phénomènes d’inondation à Gâvres (56) lors de la tempête Johanna du 10 mars 2008.

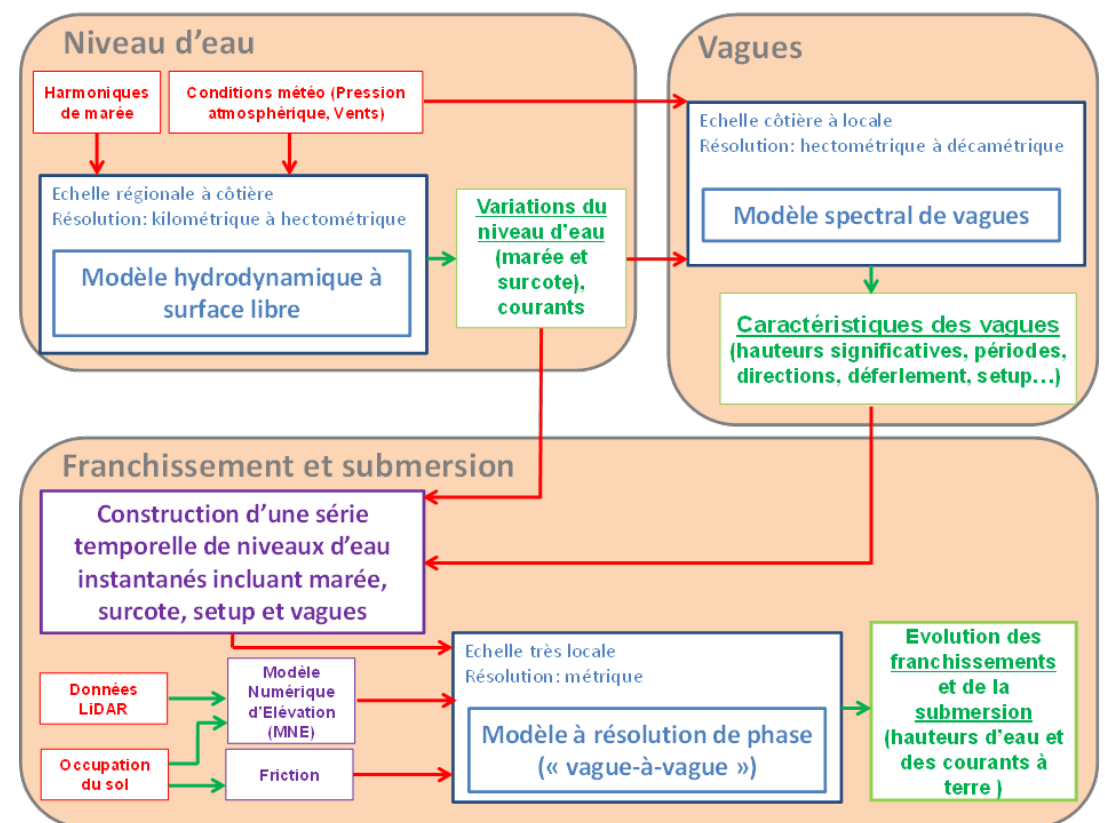

Figure 1. Méthodologie proposée pour la simulation du franchissement et de la submersion en milieu urbain. 


\section{XIII ${ }^{\text {èmes }}$ Journées Nationales Génie Côtier - Génie Civil \\ Dunkerque, 2-4 juillet 2014}

\section{Application à la submersion de Gâvres lors de la tempête Johanna}

La ville de Gâvres est située sur une presqu'île au débouché de l'estuaire du Blavet. Une ancienne zone humide polderisée et urbanisée depuis les années 50 est régulièrement affectée par des submersions marines (terrain de football et maisons environnantes), malgré la présence d'une digue en haut de la plage. La figure 2 illustre la topographie de la plage le long d'un profil (position indiquée à la figure 5) : la pente reste très régulière le long de la plage (de l'ordre de 1\%), sauf en haut de plage où elle se raidit à environ $5 \%$, avant de laisser place au plan incliné de la digue, dont la pente est de l'ordre de $22 \%$.

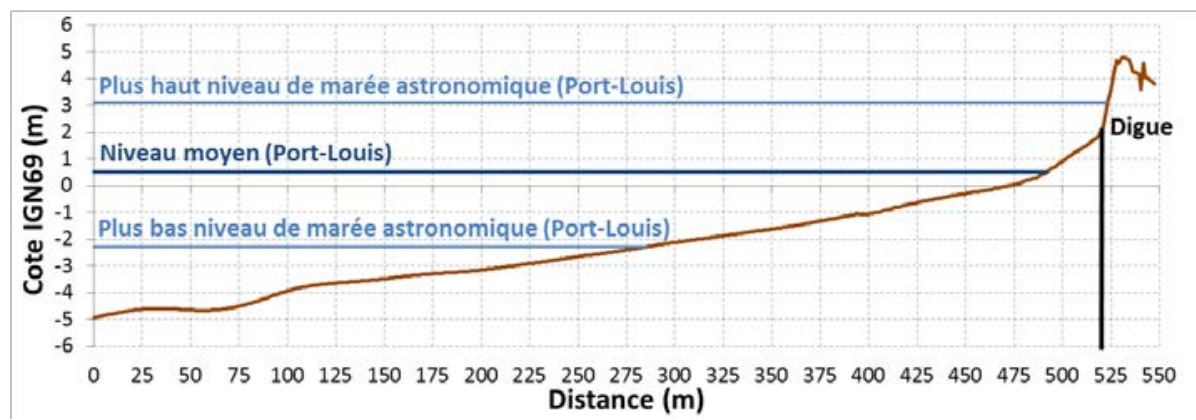

Figure 2. Profil de la Grande Plage de Gâvres au droit de la digue : les niveaux de marée indiqués correspondent à Port-Louis.

Le 10 mars 2008, lors du passage de la tempête Johanna, Gâvres a subi des submersions marines par franchissement de la digue de la Grande Plage lors des 2 pleines mers, notamment celle du matin (05h11 UTC, coefficient 106 à Port-Tudy, île de Groix), d'autant qu'un muret surmontant la digue a été en grande partie détruit sous l'effet des vagues. En effet, la tempête, tout en se déplaçant d'Ouest en Est au-dessus des îles britanniques, a essentiellement affecté la Bretagne Sud dans la matinée, puis la Bretagne Nord en fin de journée, les vents ayant tourné du Sud-Ouest au Nord-Ouest (CARIOLET et al., 2010).

\subsection{Simulation des niveaux d'eau à grande échelle}

Les niveaux d'eau, intégrant les effets cumulés de la marée et de la surcote atmosphérique, ont été simulés avec le code MARS (Ifremer), en utilisant 2 grilles de calcul emboitées (résolutions de $2 \mathrm{~km}$ sur l'Atlantique Nord-Est, et de $400 \mathrm{~m}$ sur la Bretagne Sud). Les composantes harmoniques de la marée sont issues de la base de données CST France 2005, alors que les vents et pressions atmosphériques proviennent de la base de données CFSR-NOAA. Les niveaux d'eau ont ainsi été simulés entre le 08 et le 14 mars 2008, et montrent une très bonne cohérence avec les observations des marégraphes de Port-Tudy (figure 3) et Concarneau lors de la tempête du 10 mars. 


\section{Thème 7 - Risques côtiers}

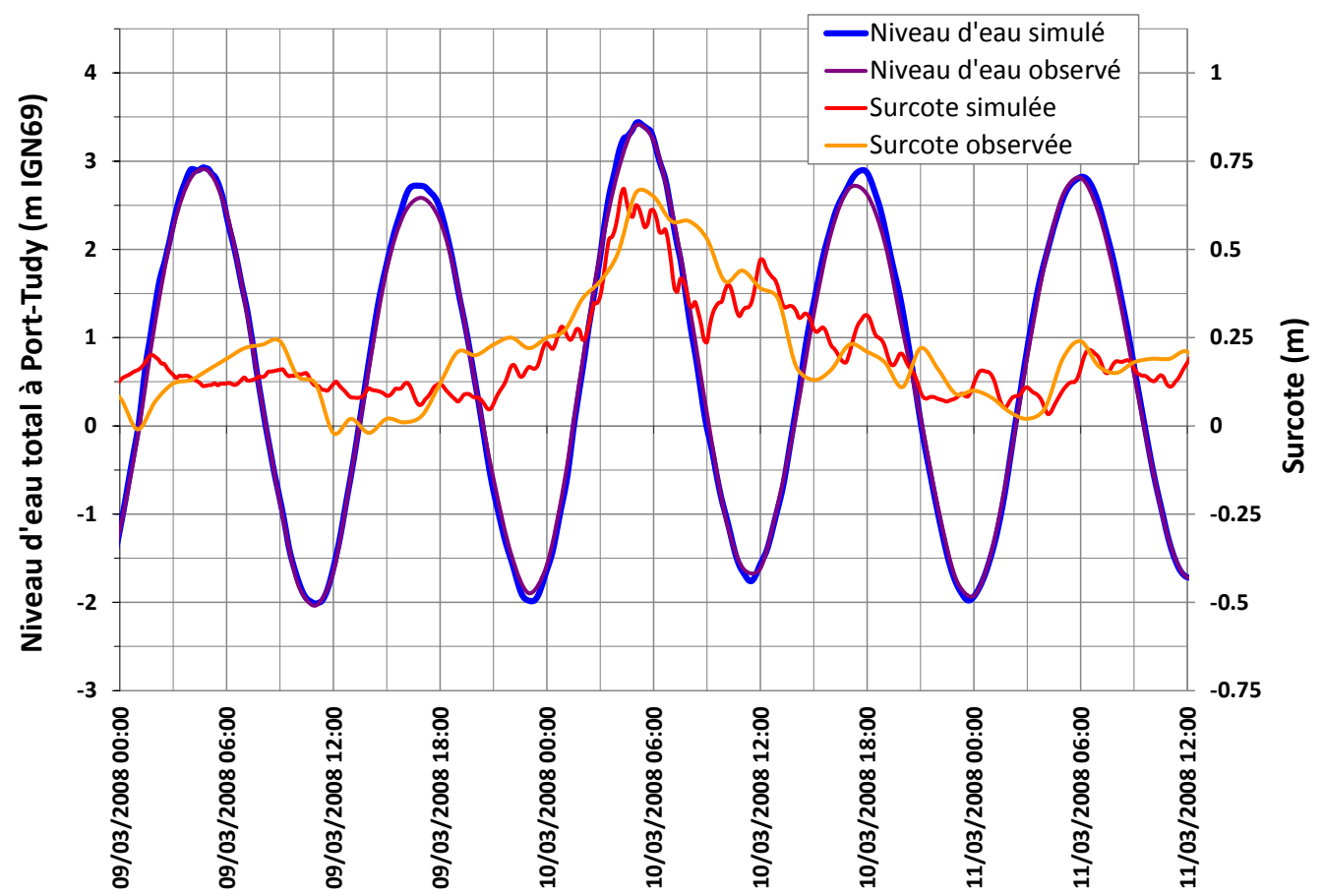

Figure 3. Validation des niveaux d'eau et surcotes simulées à Port-Tudy par rapport aux observations REFMAR (http://refmar.shom.fr/).

D’après la simulation, la surcote maximale au niveau de Gâvres a dépassé les $70 \mathrm{~cm}$ vers 04h20 UTC, se prolongeant jusqu’à 05h00 UTC. La concomitance avec la marée haute (05h20 UTC) s'est traduite à Gâvres par un niveau maximal atteint vers 05h10 UTC d'environ +3,66 m (IGN69).

\subsection{Simulation de l'état de mer}

La génération et la propagation des vagues a été simulée en 2D avec le modèle spectral SWAN sur deux grilles de calcul emboitées d'une résolution de $166 \mathrm{~m}$ sur la Bretagne Sud, et de $10 \mathrm{~m}$ au débouché de l'estuaire du Blavet. Le modèle a été forcé par les vagues simulées dans le cadre du projet IOWAGA (ARDHUIN et al., 2010), par les vents de la base de données CFSR (SAHA et al., 2010) et par les niveaux d'eau et courants issus des simulations MARS précédentes.

Les simulations de vagues, réalisées en mode instationnaire de manière à intégrer l'évolution des conditions météo-marines dans le temps, couvrent au final une période allant du 09/03/2008 (00h00) au 11/03/2008 (00h00), fournissant l'évolution des vagues en termes de spectres et de caractéristiques globales (hauteurs significatives, périodes, directions, ...), comme illustré sur la figure 4 (partie gauche). Elles permettent également d'estimer le déferlement des vagues à l'approche de la côte et le setup associé (surcote locale générée par le déferlement). 


\section{XIII ${ }^{\text {èmes }}$ Journées Nationales Génie Côtier - Génie Civil \\ Dunkerque, 2-4 juillet 2014}

Au pic de la tempête (vers 05h00), la hauteur significative des vagues au large dépasse $4 \mathrm{~m}$, et atteint encore $2 \mathrm{~m}$ sur la plage intertidale. L'analyse des résultats montre également que le déferlement des vagues se produit soit sur la plage lorsque la mer est basse, soit directement sur le plan incline de la digue à marée haute. Le niveau d'eau total peut finalement être calculé en cumulant les effets de la marée et de la surcote atmosphérique générée par la dépression et le vent (tous deux simulés à grande échelle avec le code MARS) et le setup lié au déferlement des vagues (estimé à une résolution de $10 \mathrm{~m}$ avec le code SWAN). Il s'avère que ce niveau dépasse les 3,6 m (IGN69) à la côte à Gâvres, mais reste insuffisant pour provoquer un phénomène de débordement, seul les franchissements par paquets de mer permettant d'expliquer la submersion survenue à Gâvres en 2008.

\subsection{Simulation du franchissement et la submersion}

\subsubsection{Le modèle SURF-WB}

Le code SURF-WB (MARCHE et al., 2007) est un modèle à résolution de phase ("vague à vague") issu de la dérivation, à partir des équations de Navier-Stokes homogènes et incompressibles, d'un modèle de Saint-Venant visqueux bi-dimensionnel. Il inclut des termes de diffusion, de friction, de Coriolis ou encore de tension de surface de manière rigoureuse d'un point de vue mathématique. L'utilisation de schémas numériques à capture de choc et bien équilibrés lui permet de simuler les écoulements littoraux dans le cas de fortes variations topographiques (dont le bâti), tout en convergeant vers un état stationnaire au repos et gérant les interfaces sec / mouillé et leurs évolutions de manière satisfaisante.

SURF-WB a été développé pour simuler les vagues dans les zones de surf et de swash. Toutefois, les études récentes montrent que les équations de Saint-Venant utilisées avec des schémas à capture de chocs permettent de représenter la dissipation d'énergie associée au déferlement; les modèles en cours de développement (par exemple Funwave-TVD: SHI et al. (2012); SURF-GN: TISSIER et al., 2012) utilisent d'ailleurs cette caractéristique, puisqu'ils utilisent les équations de Boussinesq au large et les équations de Saint-Venant à partir du moment où le déferlement est identifié sur la base de critères géométriques ou hydrodynamiques. Ne permettant pas cette identification, SURF-WB a été utilisé avec un forçage suffisamment proche de la côte pour que les vagues conservent un comportement réaliste.

\subsubsection{Réalisation du MNE}

Le Modèle Numérique d'Elévation (MNE) diffère du Modèle Numérique de Terrain (MNT) du fait qu'il intègre les éléments pérennes d'occupation du sol susceptibles d'intervenir avec les écoulements à terre (bâti et murs). Pour Gâvres, le MNE à haute résolution a été réalisé à partir d'acquisitions LiDAR, traitées avec l'outil LasTools 


\section{Thème 7 - Risques côtiers}

(HUG et al., 2004) et complétées par des visites de terrain. Ces données a ainsi permis d'établir une grille de calcul régulière à une résolution de $1 \mathrm{~m}$, comptant $607 \times 663$ nœuds. On peut noter que le muret surmontant la digue de la Grande Plage et partiellement détruit durant la tempête a été considéré comme endommagé dès le début des simulations.

En complément, une grille de rugosité a été élaborée, en distinguant notamment l'estran, le terrain de football et la zone urbanisée (avec de faibles coefficients de Manning caractéristiques du béton, le bâti étant par ailleurs explicitement pris en compte par le MNE).

\subsubsection{Elaboration du forçage}

Le code SURF-WB est forcé directement par une série temporelle de niveaux d'eau instantanés prenant en compte les variations du niveau d'eau et des vagues dans le temps. Cette approche est nécessaire pour reproduire au mieux la submersion marine par franchissement en contextes mésotidaux et macrotidaux. L'outil DIWASP (JOHNSON, 2002) a ainsi permis de générer une série temporelle conforme aux spectres simulés par SWAN, en prenant en compte les variations du niveau d'eau sous l'effet de la marée et de la surcote atmosphérique (modèle MARS) sur une période allant de 02h40 à 08h00 UTC le 10/03/2008, avec une modification des conditions (niveau d'eau et vagues) toutes les 10 minutes (figure 4).
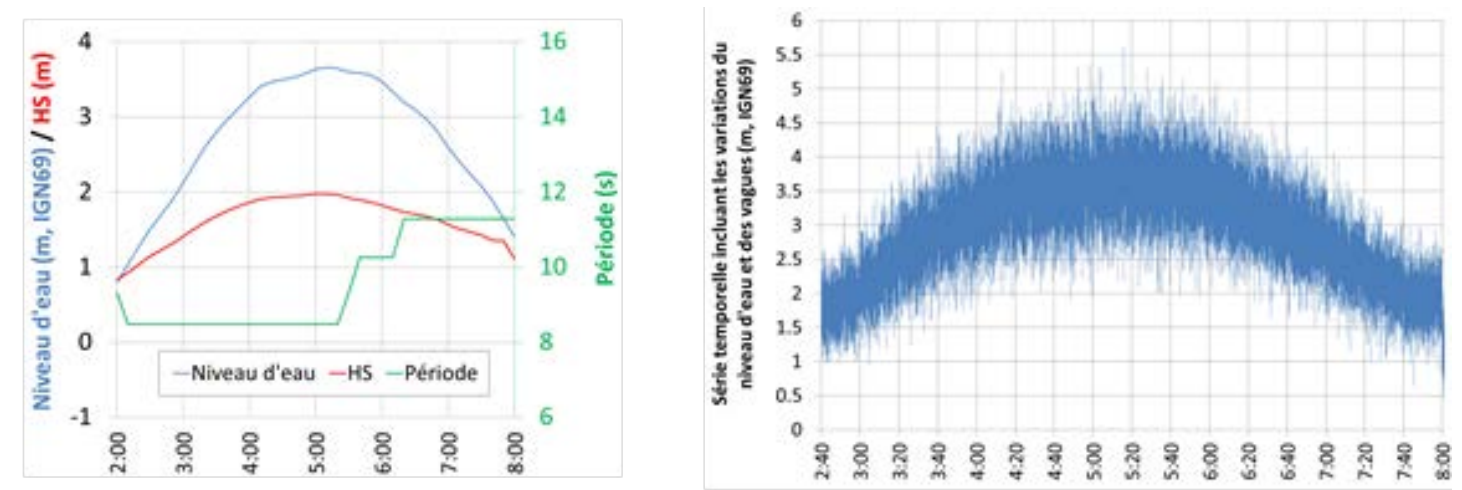

Figure 4. A gauche : Evolutions des conditions au droit de la Grande Plage de Gâvres (niveau d'eau issus du modèle MARS, vagues issues du modèle SWAN) ; A droite : Série temporelle correspondante utilisée comme forçage pour SURF-WB.

\subsection{Résultats obtenus}

Les résultats de la simulation ainsi réalisée permettent d'accéder à la fois aux franchissements par paquets de mer au cours du temps et à la submersion en résultant. 


\section{XIII ${ }^{\text {èmes }}$ Journées Nationales Génie Côtier - Génie Civil \\ Dunkerque, 2-4 juillet 2014}

\subsubsection{Validation des résultats}

Les divers éléments disponibles pour la validation reposent sur les levers effectués par CARIOLET (2010) suite à la tempête, sur les informations fournies par la mairie (zone où l'eau a stagné, maisons inondées) et sur l'étude de LE CORNEC et PEETERS (2008) (témoignage sur la chronologie, simulation de la submersion basée sur une formulation empirique du franchissement). La comparaison entre ces éléments de validation et les résultats de simulation montre une très bonne cohérence :

- les secteurs de franchissement identifiés par CARIOLET (2010) et indiqués à la figure 5 correspondent bien à l'essentiel des franchissements dans la simulation ;

- la zone de stagnation de l'eau et les maisons indiquées comme inondées par la mairie sont entièrement incluses dans la zone inondée par la simulation (figure 5) ;

- les hauteurs d'eau mesurées par CARIOLET (2010) sont en accord avec celles obtenues par la simulation, l'écart restant inférieur à $15 \mathrm{~cm}$ pour $75 \%$ des points de mesure (figure 5) ;

- la dynamique de l'inondation simulée est relativement conforme avec le témoignage disponible, malgré une légère avance sur l'arrivée de l'eau au fond de la dépression topographique (d'environ 20 minutes).

Les résultats obtenus par la simulation indiquent que les premiers franchissements apparaissent vers 03h20 UTC par la rue de la Plage et vers 03h40 par la digue. Ils s'intensifient ensuite jusqu'à 05h00, et restent significatifs jusqu'à 06h40. A terre, l'eau ruisselle vers le centre de la dépression topographique (Nord-Ouest du terrain de football) et la remplit progressivement (figure 6), jusqu'à atteindre de l'ordre de $1 \mathrm{~m}$ d'eau au maximum.
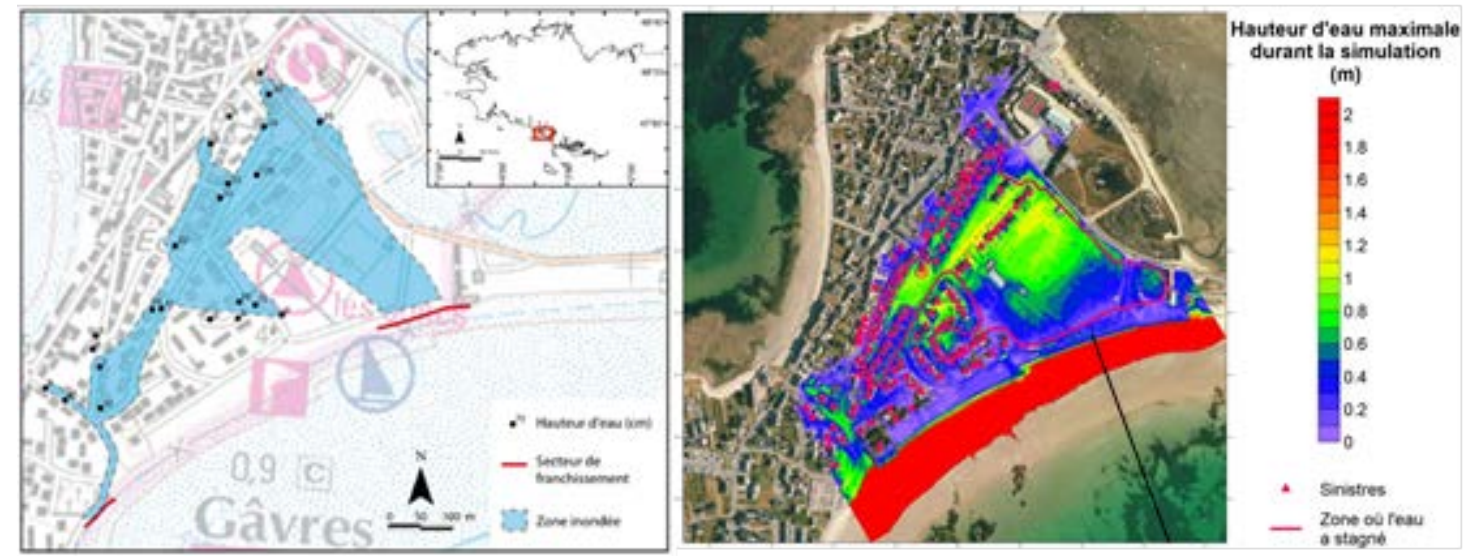

Figure 5. A gauche : Levers réalisés par CARIOLET (2010); A droite : Zone de stagnation de l'eau et maisons indiquées comme inondées par la mairie, et hauteurs d'eau maximales au cours de la simulation avec SURF-WB ; le trait noir indique la position du profil représenté à la figure 2. 


\section{Thème 7 - Risques côtiers}
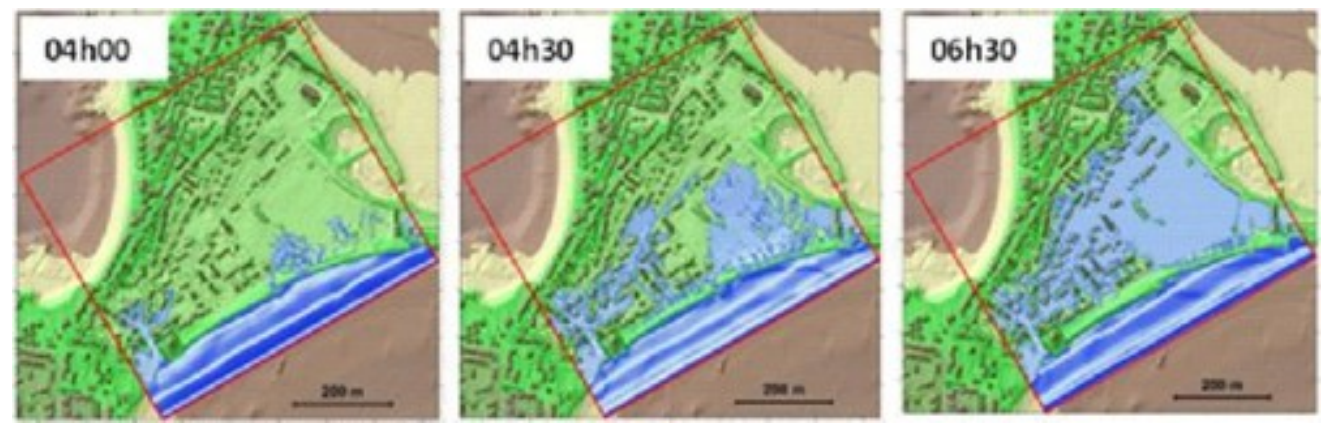

Figure 6. Aperçu de la propagation de l'inondation à 04h00, 04h30 et 06h30 (UTC).

La simulation permet également d'estimer les vitesses maximales des courants (intégrés sur la verticale) au cours de l'événement. L'utilisation d'un MNE et d'une faible rugosité en milieu urbain permet d'identifier des secteurs exposés à de forts courants, notamment dans les rues principales (rue du Parc des Sports) et en front de mer sous l'effet des franchissements. Des simulations menées dans des conditions similaires, mais sur MNT (l'urbanisation étant alors intégrée par une forte rugosité), ont permis de visualiser l'apport d'une prise en compte explicite du bâti sur les vitesses simulées. En effet, la représentation du bâti par une forte rugosité peut conduire à une large sousestimation des courants, suivant la résolution adoptée pour la rugosité. Ceci est illustré à la figure 7, qui compare les courants maximaux au cours de 2 simulations (sur MNE et sur MNT). De plus, seule l'utilisation d'un modèle vague-à-vague permet de capturer les fortes vitesses en front de mer, l'utilisation d'un débit franchissant conduisant nécessairement à un lissage des vitesses.
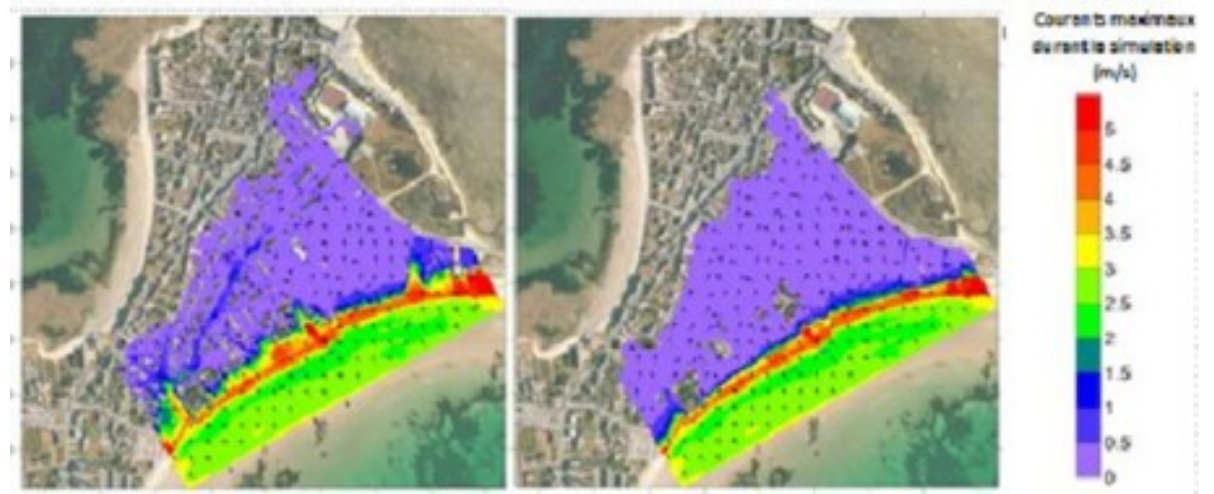

Figure 7. Courants maximaux durant la simulation sur MNE (à gauche) et sur MNT avec forte rugosité en milieu urbain (à droite).

\section{Conclusions}

La méthodologie proposée dans le présent article permet donc une simulation réaliste de la submersion par franchissement en milieu urbain: elle intègre en effet à la fois l'évolution des niveaux d'eau et de l'état de mer, le comportement de chaque vague et la 


\section{XIII ${ }^{\text {èmes }}$ Journées Nationales Génie Côtier - Génie Civil \\ Dunkerque, 2-4 juillet 2014}

propagation de la nappe d'eau à terre entre les bâtiments. Son application au cas de la submersion causée par la tempête Johanna à Gâvres (56) a ainsi permis de reconstituer de manière réaliste les événements tels qu'ils ont pu être observés sur le terrain. Cette méthodologie peut également être utilisée pour des simulations de submersion par débordement, moyennant certaines adaptations et/ou simplification (vagues négligeables, prise en compte du setup, ...).

Elle s'avère toutefois à ce jour encore difficile à mettre en œuvre, notamment pour la simulation de tous les processus physiques (déferlement, érosion, bréchification...) et compte tenu du coût en temps de calcul lorsqu'il s'agit de simuler des épisodes aussi longs dans le temps. Le développement récent et rapide des modèles numériques et la mise à disposition croissante de données topographiques à très haute résolution devraient toutefois permettre à l'avenir de généraliser ce type de simulations.

\section{Références bibliographiques}

ARDHUIN F., ROGERS A., BABANIN A., FILIPOT J.F., MAGNE R., ROLAND R., WESTHUYSEN A.V.D., QUEFFLEULOU P., LEFEVRE L., AOUF L., COLLARD F. (2010). Semi-empirical dissipation source functions for ocean waves: Part I, definition, calibration and validation. J. Phys. Oceanogr., Vol. 40, pp 1917-1941. http://dx.doi.org/10.1175/2010JPO4324.1

CARIOLET J.-M. (2010). Use of high water marks and eyewitness accounts to delineate flooded coastal areas: The case of Storm Johanna (10 March 2008) in Brittany, France. Ocean \& Coastal Management, Vol. 53, pp 679-690.

CARIOLET J.-M., COSTA S., CASPAR R., ARDHUIN F., MAGNE R., GOASGUEN G. (2010). Aspects météo-marins de la tempête du 10 mars 2008 en Atlantique et en Manche. Norois, Vol. 215, pp 11-31. http://dx.doi.org/10.4000/norois.3242

HUG C., KRZYSTEK P., FUCHSC W. (2004). Advanced LiDAR data processing with LasTools, XXth ISPRS Congress, 12-23. July 2004, Istanbul, Turkey.

JOHNSON D. (2002). DIWASP: DIrectional WAve SPectra Toolbox, Version 1.1: User Manual. Research Report No: WP 1601 DJ (V1.1), Coastal Oceanography Group Centre for Water Research, University of Western Australia, Perth.

LE CORNEC E., PEETERS P. (2008). Simulation de la tempête du 10 mars 2008 sur le site de la Grande Plage de Gâvres. Rapport d'étude GEOS-DHI.

MARCHE F., BONNETON P., FABRIE P., SEGUIN N. (2007). Evaluation of wellbalanced bore-capturing schemes for $2 d$ wetting and drying processes. International Journal for Numerical Methods in Fluids, Vol. 53, pp 867-894. http://dx.doi.org/10.1002/fld.1311

SHI F., KIRBY J.T., HARRIS J.C., GEIMAN J.D., GRILLI, S.T. (2012). A high-order adaptive time-stepping TVD solver for Boussinesq modeling of breaking waves and coastal inundation. Ocean Modelling, Vol. 43-44, pp 36-51. http://dx.doi.org/10.1016/j.ocemod.2011.12.004 
Thème 7 - Risques côtiers

TISSIER M., BONNETON P., MARCHE F., CHAZEL F., LANNES D. (2012). A new approach to handle wave breaking in fully non-linear Boussinesq models, Coastal Engineering, Vol. 67, pp 54-66. http://dx.doi.org/10.1016/j.coastaleng.2012.04.004 